\title{
Concurrent Improvement in Both Binge Eating and Depressive Symptoms with Naltrexone/ Bupropion Therapy in Overweight or Obese Subjects with Major Depressive Disorder in an Open-Label, Uncontrolled Study
}

\author{
Anna I. Guerdjikova · Brandon Walsh · Kevin Shan • \\ Amy E. Halseth · Eduardo Dunayevich · Susan L. McElroy
}

Received: May 30, 2017 / Published online: September 16, 2017

(C) The Author(s) 2017. This article is an open access publication

\section{ABSTRACT}

Introduction: Binge eating disorder (BED) is associated with obesity and major depressive disorder (MDD). Naltrexone extended-release (ER)/bupropion ER (NB) is approved as an adjunct to diet and physical activity for chronic weight management. In a prospectively designed 24-week open-label, single-arm, single-site trial of 25 women with MDD and overweight/obesity, NB reduced weight and depressive symptoms.

Methods: This post hoc analysis investigated the relationship between change in self-reported binge eating behavior (evaluated with the Binge Eating Scale [BES]) and changes in weight, control of eating, and depressive symptoms.

Enhanced content To view enhanced content for this article go to http://www.medengine.com/Redeem/ 2A0CF0603B0EEB18.

\section{A. I. Guerdjikova · S. L. McElroy}

Lindner Center of HOPE, Research Institute, Mason, $\mathrm{OH}$, USA

A. I. Guerdjikova · S. L. McElroy

Department of Psychiatry and Behavioral

Neuroscience, University of Cincinnati College of

Medicine, Cincinnati, OH, USA

B. Walsh · K. Shan · A. E. Halseth $(\bowtie)$.

E. Dunayevich

Orexigen Therapeutics, Inc., La Jolla, CA, USA

e-mail: ahalseth@orexigen.com
Results: At baseline, $91 \%$ of subjects had moderate or severe BES scores, suggesting BED. BES scores were significantly improved from week 4 , and by week $24,83 \%$ reported "little or no problem." Improvement in BES scores correlated with improvement in depressive symptoms and control of eating.

Conclusion: NB may be effective in reducing binge eating symptoms associated with MDD and overweight/obesity. Evaluation of $\mathrm{NB}$ in BED appears warranted.

Funding: Orexigen Therapeutics, Inc.

Keywords: Binge eating; Bupropion; Major depressive disorder; Naltrexone; Obesity; Psychiatry

\section{INTRODUCTION}

Binge eating disorder (BED) is a newly recognized eating disorder [1] that is associated with both obesity and major depressive disorder (MDD) [2-4]. Indeed, BED has been hypothesized to mediate, in part, the bidirectional relationship between obesity and depression [5-7].

Cognitive behavior therapy and interpersonal therapy reduce binge eating and depressive symptoms in patients with BED, but have little effect on body weight $[8,9]$. Antidepressants reduce binge eating and depressive symptoms but similarly have clinically insignificant effects on body weight [10-12]. 
Topiramate reduces binge eating and has clinically significant weight loss effects, but is difficult to tolerate because of cognitive impairment [13-15]. Sibutramine reduces binge eating, depressive symptoms, and body weight in obese patients with BED but has been removed from the market for safety reasons [16-18]. Lisdexamfetamine, approved for the treatment of moderate to severe BED in adults [19], has been shown to reduce binge eating and body weight in patients with BED, but the presence of clinically significant depressive symptoms was a specific exclusionary criterion [20-22]. New treatments for BED, especially for individuals with concomitant MDD and obesity, are greatly needed.

Naltrexone extended-release (ER) and bupropion ER combination (NB) is an anti-obesity agent approved in the USA, European Union, and South Korea as an adjunct to diet and physical activity for chronic weight management in obese adults or overweight adults with one or more weight-related co-morbidities. NB is believed to reduce appetite through sustained activation of hypothalamic pro-opiomelanocortin (POMC) neurons and to reduce cravings through actions in the mesolimbic reward system [23, 24]. A prospectively designed open-label, single-arm, single-site 24-week exploratory study of NB in overweight or obese subjects with MDD showed that $\mathrm{NB}$, in combination with dietary and behavioral intervention, was associated with significant reduction of depressive symptoms and of body weight from baseline $(-9.2 \%$ at 24 weeks in observed subjects) as well as improvement in ratings of control of eating [25]. The Binge Eating Scale [26] (BES), used as a measure of self-assessed binge eating behavior and cognition, was a secondary outcome in the study. BES scores were significantly decreased during the study. In the current investigation, the effect of NB on binge eating symptomatology was evaluated with an exploratory analysis of the relationships between change in BES score and change in depression scores (using the Montgomery-Åsberg Depression Rating Scale [MADRS] and Inventory of Depressive Symptomatology-Self-Report [IDS-SR]) [27, 28], change in weight, and change in self-reported difficulty in controlling eating, assessed using the Control of Eating Questionnaire [CoEQ [29]]).

\section{METHODS}

The study design, inclusion/exclusion criteria, and analysis population for this open-label study have been described previously [25]. Briefly, a single-center, open-label, single-arm 24-week prospective study evaluated combination therapy with $32 \mathrm{mg} /$ day naltrexone ER and $360 \mathrm{mg} /$ day bupropion ER (NB) in overweight/ obese subjects with MDD. Medication was initiated at one-quarter of the daily maintenance dose and escalated over the first 4 weeks of treatment. Previously reported key inclusion criteria included age 18-65 years; body mass index (BMI) $\geq 27$ to $\leq 43 \mathrm{~kg} / \mathrm{m}^{2}$; MDD, without psychotic features, according to DSM-IV criteria; and IDS-SR score $>26$ at screening. The presence of BED was not a selection criterion; lifetime history or anorexia nervosa or bulimia were exclusionary. Psychotropic medications, other than low dose benzodiazepines or hypnotic agents for the treatment of insomnia, were prohibited. Body weight and MADRS were assessed at weeks $1,2,3,4,6,8,12,16,20$, and 24; IDS-SR was assessed at weeks $4,8,12,16,20$, and 24. BES and CoEQ were assessed at weeks 4 , 8,12 , and 24 . All subjects received dietary and behavioral counseling at baseline and weeks 6 , 12 , and 16 and a prescription for exercise from a registered dietician. The primary efficacy endpoint of this study was the change in MADRS total score from baseline to week 12 [25]. Key secondary endpoints included change in MADRS total score from baseline to week 24, as well as change in body weight, IDS-SR total score, CoEQ scores, and BES scores from baseline to weeks 12 and 24 [25]. The study protocol was approved by the University of Cincinnati institutional review board, and each patient provided written informed consent prior to study entry. Study conduct was consistent with Good Clinical Practice standards and the Helsinki Declaration of 1964, as revised in 2013. This article is based on analysis of the data from a previously conducted study (ClinicalTrials.gov 
Identifier NCT00624858) and does not involve any new studies of human or animal subjects performed by any of the authors.

For the present exploratory analysis, change in BES total score at weeks $4,8,12$, and 24 was investigated, along with the relationships between the change in BES total score and the change in MADRS total score, IDS-SR total score, and difficulty controlling eating based on CoEQ Question 19 (CoEQ 19) score. The BES consists of 16 self-reported weighted items (score $0-3$ each) designed to assess binge eating problems in obese individuals by appraising both the behavioral manifestations of, and cognition surrounding, a binge episode. A total BES score $\leq 17$ is interpreted as "little or no problem," $18-26$ as a "moderate problem," and $\geq 27$ as a "severe problem." The CoEQ consists of 20 subject-reported questions, each scored with a $100-\mathrm{mm}$ visual analog scale, designed to assess various aspects of appetite, food craving, eating behavior, and mood [29]. CoEQ 19 reads: "Generally, how difficult has it been to control your eating?"

For these analyses, only observed data from the full analysis set (all subjects who had a baseline measurement, and had at least one post-baseline MADRS total score measurement while on study drug) were used. For the categorical outcome variables, descriptive statistics included number and percentage of subjects for each category. Percentages were calculated on the basis of subjects with non-missing data at a given time point as the denominator (i.e., those subjects with missing data were excluded). For the continuous outcome variables, descriptive statistics included number of subjects $(n)$, mean, standard deviation (SD), median, minimum, and maximum. Within-group $t$ test statistics and 95\% confidence intervals were calculated to evaluate whether the change from baseline was statistically significantly different from zero, without adjustment for multiplicity. Correlations between BES score change and IDS-SR, MADRS, CoEQ 19, or weight change were evaluated using Pearson's correlation coefficient. The significance level was defined as $p<0.05$ and all reported $p$ values are 2-sided. All statistical analyses were performed using $\mathrm{SAS}^{\circledR}$ software (Version 9.1 or
Later, Copyright (C) 2002-2010, SAS Institute Inc., Cary, NC).

\section{RESULTS}

As previously reported, all 25 participants were women, of which 23 were included in the full analysis set presented in this analysis. At baseline $(n=23)$ mean (SD) BMI was $35.3(4.2) \mathrm{kg} /$ $\mathrm{m}^{2}$, and mean (SD) scores for the measurement tools used in these analyses were MADRS 23.7 (4.2), IDS-SR 43.7 (8.1), BES 28.7 (8.7), and CoEQ 1980.2 (16.4). Moderate or severe problems with binge eating (per BES) were present at baseline in $91 \%$ of subjects (Fig. 1). Twelve subjects discontinued treatment prior to week 24: 1 lost to follow-up, 1 due to non-compliance with study drug, and 10 due to adverse events (all single events, none of which were serious) [25]. NB was associated with significant reductions from baseline in body weight and MADRS, IDS-SR, and CoEQ 19 scores that were observed as early as week 1 for MADRS and body weight [25] and week 4 for IDS-SR and CoEQ 19 (Table 1); these improvements were all sustained through study end. As presented in Fig. 1, BES scores were significantly improved at the first post-baseline measurement (week 4; $n=17$ ) and improvement was maintained throughout the trial. By week $8(n=16)$, no subjects exhibited "severe" BES scores, and by week $24(n=12), 83 \%$ of subjects exhibited "little or no problem" per the BES (Fig. 1).

A significant correlation was observed between improvement in IDS-SR and BES scores at all time points $(r=0.59-0.70$; all $p<0.05$; Fig. 2). MADRS change also demonstrated a significant relationship with BES score change at weeks $4(r=0.66, p<0.01)$ and $12(r=0.54$, $p<0.05)$, and while a similar trend was observed at weeks 8 and 24, the relationship did not achieve statistical significance at those time points $(r=0.45, p=0.08$ at week $8 ; r=0.43$, $p=0.16$ at week 24; Fig. 3). CoEq 19 was significantly correlated with change in BES scores at week $4(r=0.74 ; p<0.001)$ and week 24 $(r=0.73 ; p<0.01)$, but did not achieve statistical significance at weeks 8 and $12(r=0.48$ to $0.51 ; p=0.05$ to 0.08$)$. Weight loss was not 

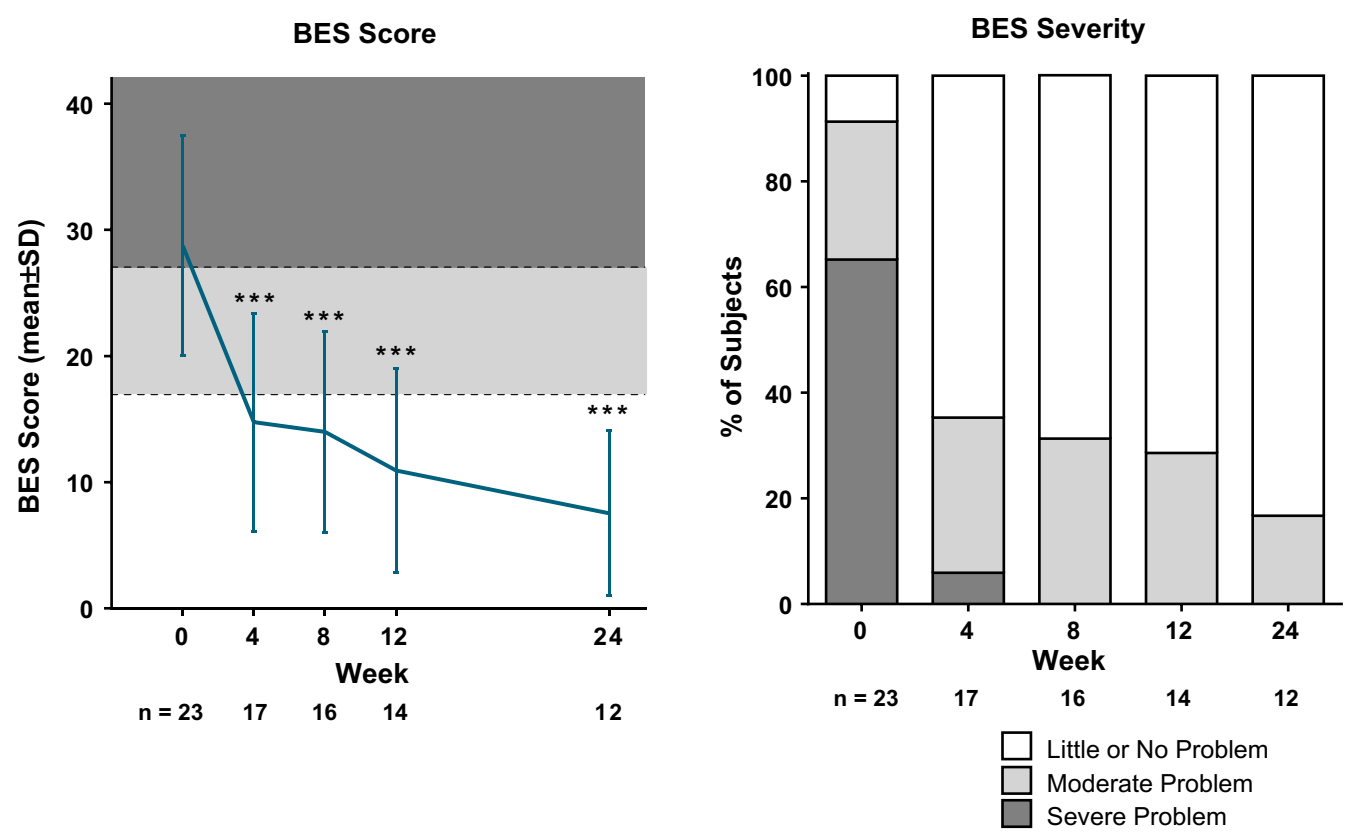

Fig. 1 BES score and BES severity. BES Binge Eating Scale, SD standard deviation. Data from the observed population. ${ }^{* * *} p<0.001$ vs. baseline

Table 1 Change from baseline in body weight and measures of depression and difficulty controlling eating

\begin{tabular}{lllll}
\hline & Weight & MADRS & IDS-SR & CoEQ 19 \\
\hline Baseline $(n=23)$ & $96.7 \mathrm{~kg}(90.3,103.1)$ & $23.7(21.9,25.5)$ & $43.7(40.2,47.2)$ & $80.2(n=23,16.4)$ \\
Week 4 $(n=20)$ & $-2.3 \%(-3.3,-1.3)^{* * *}$ & $-12.0(-15.1,-8.9)^{* * *}$ & $-15.8(-21.3,-10.4)^{* * *}$ & $-40.2(-54.3,-26.2)^{* * *}$ \\
Week 8 $(n=17)$ & $-3.6 \%(-5.4,-1.9)^{* * *}$ & $-13.7(-16.2,-11.1)^{* * *}$ & $-19.6(-24.6,-14.5)^{* * *}$ & $-41.5(-55.4,-27.7)^{* * *}$ \\
Week 12 $(n=14)$ & $-6.1 \%(-8.8,-3.4)^{* * *}$ & $-14.6(-17.8,-11.3)^{* * *}$ & $-20.1(-27.3,-12.9)^{* * *}$ & $-44.4(-60.8,-27.9)^{* * *}$ \\
Week 24 $(n=13)$ & $-9.2 \%(-12.9,-5.4)^{* * *}$ & $-19.1(-22.4,-15.7)^{* * *}$ & $-26.9(-35.2,-18.7)^{* * *}$ & $-41.8(-59.2,-24.5)^{* * *}$ \\
\hline
\end{tabular}

MADRS Montgomery-Åsberg Depression Rating Scale, IDS-SR Inventory of Depressive Symptomatology-Self-Report, CoEQ 19 Control of Eating Questionnaire Question 19

Data are mean $(95 \%$ confidence interval)

${ }^{* * *} p<0.001$ vs. baseline

correlated with BES score change at any time point. As previously reported [25], the most common adverse events reported during the study were nausea $(n=12 ; 48 \%)$, constipation $(n=8 ; 32 \%)$, headache $(n=8 ; 32 \%)$, insomnia $(n=8 ; 32 \%)$, dizziness $(n=7 ; 28 \%)$, and hot flush $(n=7 ; 28 \%)$, the majority of which were considered moderate in severity. Because NB includes an antidepressant as one of its components, it carries a boxed warning common to the class regarding suicidal thought and behavior in children, adolescents, and young adults age 24 and under. No adverse events related to suicidal thoughts or actions were observed in this study. Bupropion and NB have a dose-related potential to increase the risk of seizure, which is believed to have an incidence of approximately $0.1 \%$, and be increased in patients with bulimia or anorexia. No seizures were reported in this study.

\section{DISCUSSION}

In this prospectively designed open-label study of the treatment of participants with MDD and overweight/obesity, NB, given in conjunction with dietary and physical activity counseling, was associated with a significant decrease in binge eating score, as well as improvements in 

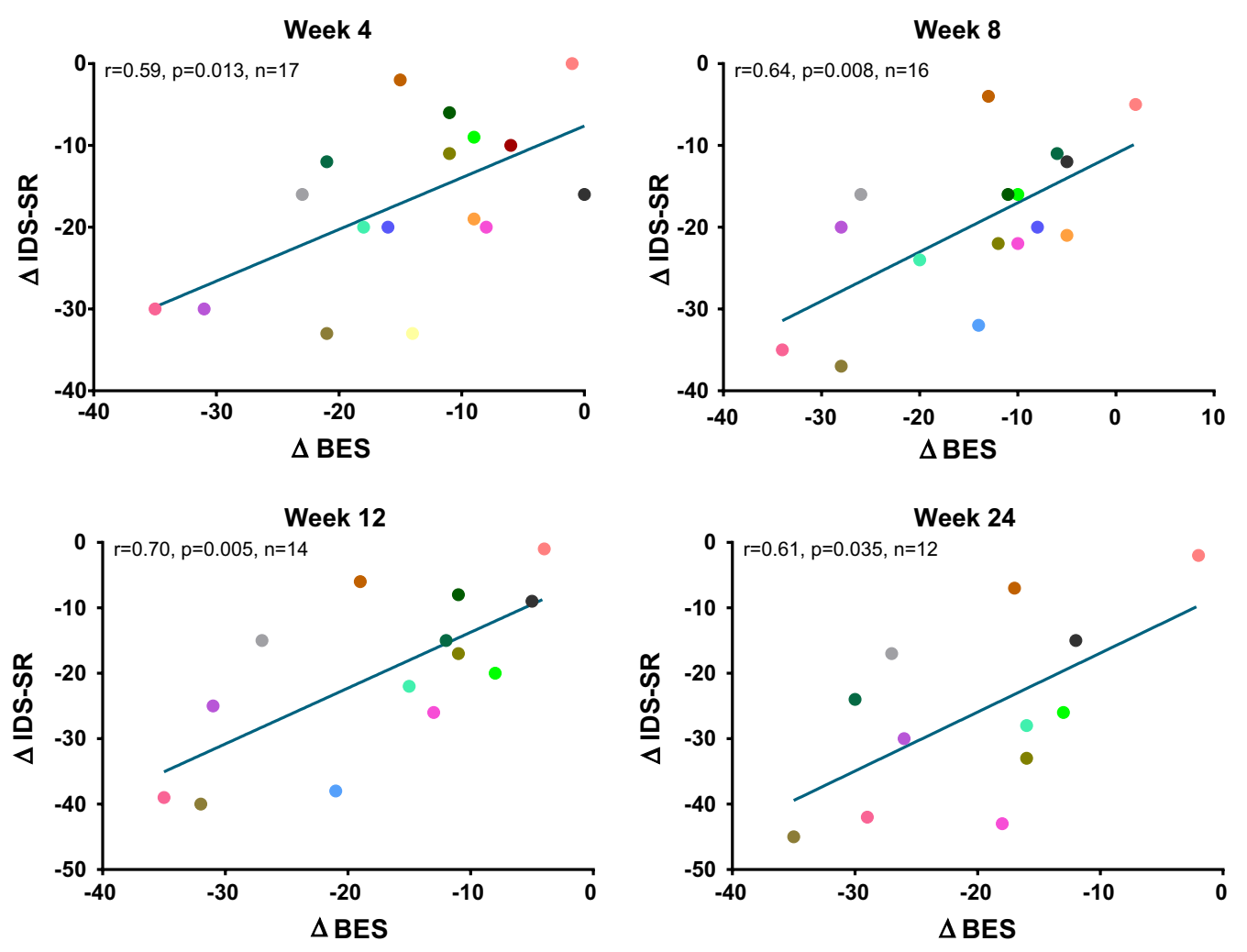

Fig. 2 Change in BES score vs. change in IDS-SR. IDS-SR Inventory of Depressive Symptomatology-Self-Report, BES Binge Eating Scale

depressive symptoms, body weight, and control over eating. BED was not an entry criterion, and although BES scores do not necessarily equate with a formal BED diagnosis, it is noteworthy that BES scores were unexpectedly high and clinically significant at baseline: $91 \%$ of subjects had moderate or severe binge eating behavior according to this scale. From week 8 onward, no patient continued to exhibit BES scores in the severe range. Moreover, improvement in binge eating symptoms generally correlated with improvement in depressive symptoms based on both clinician-scored (MADRS) and self-reported (IDS-SR) depressive scores (although the correlations were not significant at each time point using the MADRS), and control over eating. These findings suggest that binge eating, at least when accompanied by MDD and overweight/obesity, may respond to $\mathrm{NB}$ plus behavioral treatment.

In prior small studies, bupropion monotherapy has been shown to reduce binge eating in bulimia nervosa (BN) [30] but not BED [31], where it was associated with a small but statistically significant amount of weight loss. Studies of opioid antagonist monotherapy have been mixed but largely negative in both $\mathrm{BN}$ and BED [32]. The present findings raise the question whether binge eating, in particular when present in individuals with MDD, might respond better to $\mathrm{NB}$ than to bupropion alone or naltrexone alone, as has been shown for weight loss in people with obesity. Randomized controlled trials are needed to determine whether true BED responds better to NB than to its individual components.

$\mathrm{NB}$ is postulated to affect both the homeostatic and reward centers of the brain [23], and the improvement in self-reported binge behavior assessed with the BES observed in this study may, in part, be related to the purported effect of NB on improving measures of control of eating and cravings [23, 33-37]. However, given the complex bidirectional associations between 

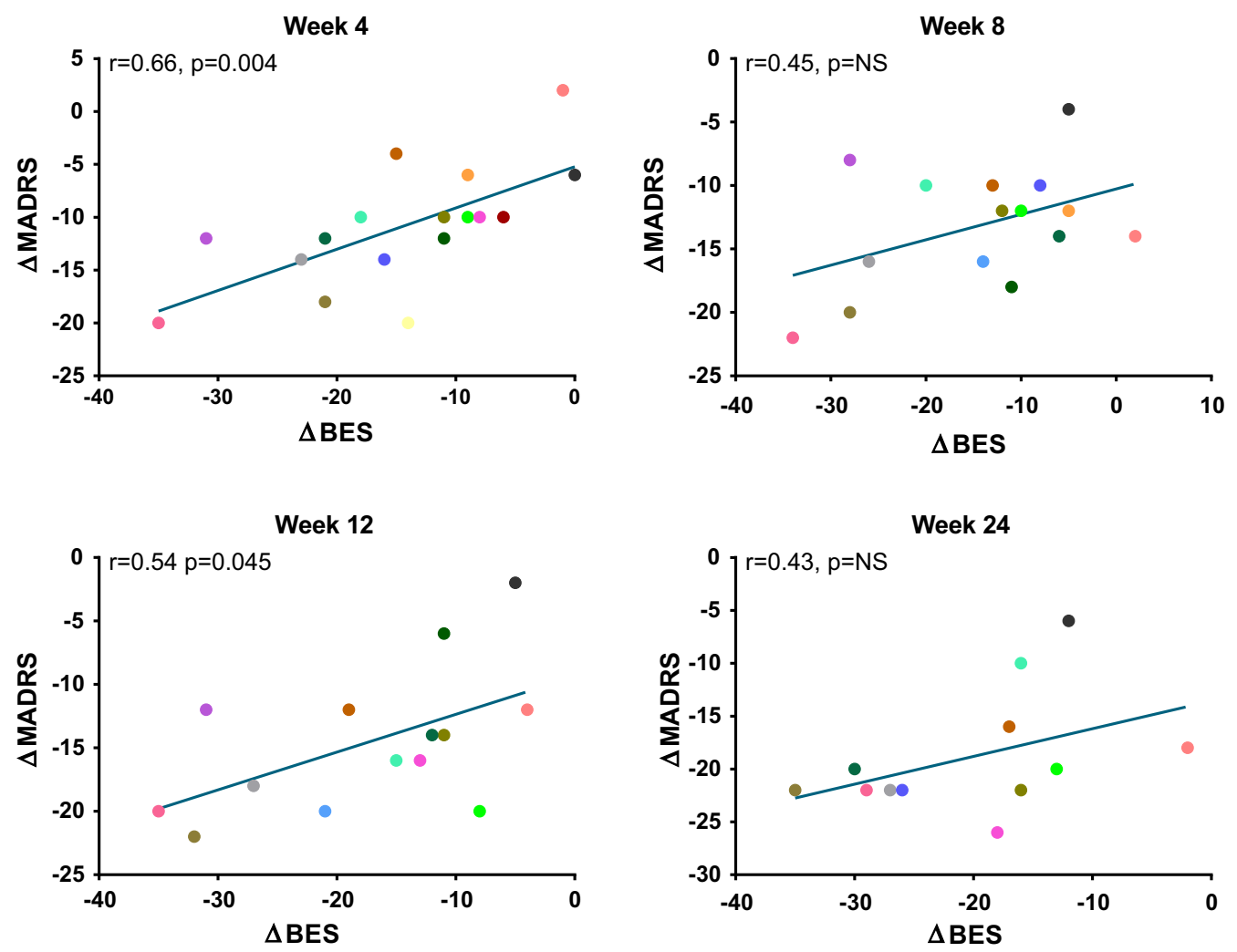

Fig. 3 Change in BES score vs. change in MADRS. MADRS Montgomery-Åsberg Depression Rating Scale, BES Binge Eating Scale

mood, eating behavior, and body weight, as well as the uncontrolled design of the study, it is difficult to ascertain whether these improvements are primarily related to the effects of NB on control over eating, other mechanisms of weight loss with NB, the antidepressant effects of bupropion, the counseling received by subjects, or other factors related to clinical trial participation. Finally, although there was no association between change in BES score and weight loss, it should be noted that weight change is a gradual process and temporally dissociated from changes in eating behavior and mood. Thus, longer-term evaluation of the relationship between these outcomes may be warranted.

Limitations of this study include its open-label, single-site (tertiary care center) design, lack of control group, small sample size, and high dropout rate, and the fact that all participants were women. Thus, while these results are promising, limited inferences can be made to the broader MDD or BED obese population. The possibility that subjects' binge eating behavior responded to dietary and physical activity counseling rather than NB cannot be excluded. Similarly, the contribution of the behavioral counseling to the change in body weight cannot be determined, although in previous studies, mean weight loss in participants who complete 1 year of treatment with NB tends to be approximately $4-6 \%$ greater than in study participants receiving the identical instruction on diet and exercise [33-36]. It is also unknown if these findings using the BES would generalize to patients with diagnosed BED, to men with BED, or to people with BED without co-occurring MDD or overweight/ obesity.

\section{CONCLUSION}

These findings suggest that binge eating, at least when accompanied by MDD and overweight/ obesity, may respond to NB plus behavioral 
treatment. Evaluation of NB in BED in randomized controlled trials appears warranted.

\section{ACKNOWLEDGEMENTS}

The study that provided data for this analysis, the article processing charges, and the Open Access fee were funded by Orexigen Therapeutics, Inc. Editorial support was provided by Dewey McLin III, PhD of Orexigen Therapeutics, Inc. All authors had full access to all of the data in this study and take complete responsibility for the integrity of the data and accuracy of the data analysis. These data were presented at Obesity Week 2013, November 15, Atlanta, GA. All named authors meet the International Committee of Medical Journal Editors (ICMJE) criteria for authorship for this manuscript, take responsibility for the integrity of the work as a whole, and have given final approval for the version to be published.

Disclosures. Anna I. Guerdjikova is employed by University of Cincinnati and Lindner Center of HOPE. She is a consultant to or member of the scientific advisory boards of Avanir and Bracket Inc. Eduardo Dunayevich is a full-time Takeda employee, and former employee of Orexigen, but holds no stock or compensation from Orexigen. Susan McElroy is a consultant to or member of the scientific advisory boards of Avanir, Bracket, F. Hoffmann-La Roche Ltd., Ironshore, Mitsubishi Tanabe Pharma America, Myriad, Novo Nordisk, Otsuka, Shire, and Sunovion. She is a principal or co-investigator on studies sponsored by Allergan, Azevan, Brainsway, Marriott Foundation, Medibio, Myriad, National Institute of Mental Health, Novo Nordisk, Shire, and Sunovion. She has been a principal investigator on a study supported by Orexigen Therapeutics. She is also an inventor on US Patent No. 6323,236 B2 (Use of sulfamate derivatives for treating impulse control disorders), and along with the patent's assignee, University of Cincinnati, Cincinnati, Ohio, has received payments from Johnson \& Johnson, which has exclusive rights under the patent. Brandon
Walsh is a current employee of Elcelyx Therapeutics, Inc., and a former employee of and current shareholder of Orexigen. Amy Halseth and Kevin Shan are current employees and shareholders of Orexigen.

Compliance with Ethics Guidelines. The study protocol was approved by the University of Cincinnati institutional review board, and each patient provided written informed consent prior to study entry. Study conduct was consistent with Good Clinical Practice standards and the Helsinki Declaration of 1964 , as revised in 2013. This article is based on analysis of the data from a previously conducted study (ClinicalTrials.gov Identifier NCT00624858) and does not involve any new studies of human or animal subjects performed by any of the authors.

Data availability. The datasets generated during and/or analyzed during the current study are available from the corresponding author on reasonable request.

Open Access. This article is distributed under the terms of the Creative Commons Attribution-NonCommercial 4.0 International License (http://creativecommons.org/licenses/ by-nc/4.0/), which permits any noncommercial use, distribution, and reproduction in any medium, provided you give appropriate credit to the original author(s) and the source, provide a link to the Creative Commons license, and indicate if changes were made.

\section{REFERENCES}

1. APA: diagnostic and statistical manual of mental disorders, 5th ed. Washington DC: American Psychiatric Association, 2013.

2. Hudson JI, Hiripi E, Pope HG Jr, Kessler RC. The prevalence and correlates of eating disorders in the national comorbidity survey replication. Biol Psychiatry. 2007;61:348-58.

3. Javaras KN, Pope HG, Lalonde JK, et al. Co-occurrence of binge eating disorder with psychiatric and medical disorders. J Clin Psychiatry. 2008;69:266-73. 
4. Kessler RC, Berglund PA, Chiu WT, et al. The prevalence and correlates of binge eating disorder in the World Health Organization World Mental Health Surveys. Biol Psychiatry. 2013;73:904-14.

5. de Wit L, Luppino F, van Straten A, Penninx B, Zitman F, Cuijpers P. Depression and obesity: a meta-analysis of community-based studies. Psychiatry Res. 2010;178:230-235.

6. Luppino FS, de Wit LM, Bouvy PF, et al. Overweight, obesity, and depression: a systematic review and meta-analysis of longitudinal studies. Arch Gen Psychiatry. 2010;67:220-9.

7. McElroy SL, Kotwal R, Malhotra S, Nelson EB, Keck PE, Nemeroff CB. Are mood disorders and obesity related? A review for the mental health professional. J Clin Psychiatry. 2004;65:634-51 (quiz 730).

8. Iacovino JM, Gredysa DM, Altman M, Wilfley DE. Psychological treatments for binge eating disorder. Curr Psychiatry Rep. 2012;14:432-46.

9. Wilson GT, Wilfley DE, Agras WS, Bryson SW. Psychological treatments of binge eating disorder. Arch Gen Psychiatry. 2010;67:94-101.

10. Aigner M, Treasure J, Kaye W, Kasper S. World Federation of Societies of Biological Psychiatry (WFSBP) guidelines for the pharmacological treatment of eating disorders. World J Biol Psychiatry. 2011;12:400-43.

11. Guerdjikova AI, McElroy SL, Winstanley EL, et al. Duloxetine in the treatment of binge eating disorder with depressive disorders: a placebo-controlled trial. Int J Eat Disord. 2012;45:281-9.

12. Stefano SC, Bacaltchuk J, Blay SL, Appolinario JC. Antidepressants in short-term treatment of binge eating disorder: systematic review and meta-analysis. Eat Behav. 2008;9:129-36.

13. McElroy SL, Arnold LM, Shapira NA, et al. Topiramate in the treatment of binge eating disorder associated with obesity: a randomized, placebo-controlled trial. Am J Psychiatry. 2003;160:255-61.

14. McElroy SL, Hudson JI, Capece JA, Beyers K, Fisher $\mathrm{AC}$, Rosenthal NR. Topiramate for the treatment of binge eating disorder associated with obesity: a placebo-controlled study. Biol Psychiatry. 2007;61:1039-48.

15. McElroy SL, Shapira NA, Arnold LM, et al. Topiramate in the long-term treatment of binge-eating disorder associated with obesity. J Clin Psychiatry. 2004;65:1463-9.
16. Appolinario JC, Bacaltchuk J, Sichieri R, et al. A randomized, double-blind, placebo-controlled study of sibutramine in the treatment of binge-eating disorder. Arch Gen Psychiatry. 2003;60:1109-16.

17. Milano W, Petrella C, Casella A, Capasso A, Carrino S, Milano L. Use of sibutramine, an inhibitor of the reuptake of serotonin and noradrenaline, in the treatment of binge eating disorder: a placebo-controlled study. Adv Ther. 2005;22:25-31.

18. Wilfley DE, Crow SJ, Hudson JI, et al. Efficacy of sibutramine for the treatment of binge eating disorder: a randomized multicenter placebo-controlled double-blind study. Am J Psychiatry. 2008;165:51-8.

19. Vyvanse [package insert]. Lexington, MA: Shire US; 2017.

20. McElroy SL, Hudson JI, Mitchell JE, et al. Efficacy and safety of lisdexamfetamine for treatment of adults with moderate to severe binge-eating disorder: a randomized clinical trial. JAMA Psychiatry. 2015;72:235-46.

21. McElroy SL, Hudson J, Ferreira-Cornwell MC, et al. Lisdexamfetamine dimesylate for adults with moderate to severe binge eating disorder: results of two pivotal phase 3 randomized controlled trials. Neuropsychopharmacology. 2015;41:1251-60.

22. Gasior M, Hudson J, Quintero J, Ferreira-Cornwell MC, Radewonuk J, McElroy SL. A phase 3, multicenter, open-label, 12-month extension safety and tolerability trial of lisdexamfetamine dimesylate in adults with binge eating disorder. J Clin Psychopharmacol. 2017;37(3):315-22.

23. Billes SK, Sinnayah P, Cowley MA. Naltrexone/ bupropion for obesity: an investigational combination pharmacotherapy for weight loss. Pharmacol Res. 2014;84:1-11.

24. Greenway FL, Whitehouse MJ, Guttadauria M, et al. Rational design of a combination medication for the treatment of obesity. Obesity (Silver Spring). 2009;17:30-9.

25. McElroy SL, Guerdjikova AI, Kim DD, et al. Naltrexone/bupropion combination therapy in overweight or obese patients with major depressive disorder: results of a pilot study. Prim Care Companion CNS Disord 2013. doi:10.4088/PCC. $12 \mathrm{~m} 01494$.

26. Gormally J, Black S, Daston S, Rardin D. The assessment of binge eating severity among obese persons. Addict Behav. 1982;7:47-55. 
27. Montgomery SA, Asberg M. A new depression scale designed to be sensitive to change. Br J Psychiatry. 1979;134:382-9.

28. Rush AJ, Gullion CM, Basco MR, Jarrett RB, Trivedi $\mathrm{MH}$. The inventory of depressive symptomatology (IDS): psychometric properties. Psychol Med. 1996;26:477-86.

29. Hill AJ, Weaver CF, Blundell JE. Food craving, dietary restraint and mood. Appetite. 1991;17:187-97.

30. Horne RL, Ferguson JM, Pope HG Jr, et al. Treatment of bulimia with bupropion: a multicenter controlled trial. J Clin Psychiatry. 1988;49:262-6.

31. White M, Grilo C. Bupropion for overweight women with binge-eating disorder: a randomized, double-blind, placebo-controlled trial. J Clin Psychiatry. 2013;74:400-6.

32. McElroy SL, Guerdjikova AI, Blom TJ, et al. A placebo-controlled pilot study of the novel opioid receptor antagonist ALKS-33 in binge eating disorder. Int J Eat Disord. 2013;46:239-45.

33. Apovian CM, Aronne L, Rubino D, et al. Group C-IS: a randomized, phase 3 trial of naltrexone SR/ bupropion SR on weight and obesity-related risk factors (COR-II). Obesity. 2013;21:935-43.

34. Greenway FL, Fujioka K, Plodkowski RA, et al. Group C-IS: effect of naltrexone plus bupropion on weight loss in overweight and obese adults (COR-I): A multicentre, randomised, double-blind, placebo-controlled, phase 3 trial. Lancet. 2010;376:595-605.

35. Hollander P, Gupta AK, Plodkowski R, et al. Group CO-DS: effects of naltrexone sustained-release/ bupropion sustained-release combination therapy on body weight and glycemic parameters in overweight and obese patients with type 2 diabetes. Diabetes Care. 2013;36:4022-9.

36. Wadden TA, Foreyt JP, Foster GD, et al. Weight loss with naltrexone SR/bupropion SR combination therapy as an adjunct to behavior modification: the COR-BMOD trial. Obesity. 2011;19:110-20.

37. Dalton M, Finlayson G, Walsh B, Halseth AE, Duarte C, Blundell JE. Early improvement in food cravings are associated with long-term weight loss success in a large clinical sample. Int J Obes (Lond). 2017. doi:10.1038/ijo.2017.89. 